14.1

\title{
Модель чередующегося баллистического броуновского переноса частиц и асимптотическое приближение к ней
}

\author{
(C) С.А. Руколайне \\ Физико-технический институт им. А. Ф. Иоффре, \\ 194021 Санкт-Петербург, Россия \\ e-mail: rukol@ammp.ioffe.ru
}

(Поступило в Редакцию 25 декабря 2017 г.)

\begin{abstract}
Предложена модель чередующегося переноса (движения) частиц, молекул биологических особей. При перемещении в пространстве частицы могут находиться в одной из двух фаз: в баллистической (активной), когда частица движется с постоянной скоростью в некотором направлении, и в броуновской (пассивной), когда частица находится в броуновском движении. Частица может случайным образом переходить от одной фазы движения к другой, и наоборот, причем длительность пассивной фазы имеет экспоненциальное распределение, а длительность активной фазы (длина свободного пробега частицы) распределена по произвольному закону. Найдено асимптотическое приближение к рассматриваемой модели в предположении, переходы между фазами движения происходят очень часто. Приведен пример, демонстрирующий, что неверное представление о распределении длины свободного пробега частиц может приводить к значительной ошибке.
\end{abstract}

DOI: 10.21883/JTF.2018.09.46413.2615

\section{Введение}

Чередующийся перенос биологических объектов, включающий направленное и броуновское движения, встречается в самом различном виде и масштабах: от внутриклеточного транспорта грузов (органелл, везикул и др.) по сети микротрубочек и актиновых нитей (по цитоскелету) [1] до поиска животными пищи [2,3]. Различные модели чередующегося переноса представлены в [2,4-10] В этих моделях, кроме [8], распределение длины свободного пробега частиц экспоненциально. В [8] рассматривается модель, комбинирующая броуновское движение и случайные блуждания Леви (Lévy walks), но оценивается лишь среднеквадратичное отклонение траекторий. Однако предположение об экспоненциальном распределении длины свободного пробега частиц справедливо далеко не всегда [3,11-13]

В настоящей работе предложена модель чередующегося переноса (движения) частиц, молекул или биологических особей. При перемещении в пространстве частицы могут находиться в одной из двух фаз: в баллистической (активной), когда частица движется с постоянной скоростью в некотором направлении, и в броуновской (пассивной), когда частица находится в броуновском движении. Частица может случайным образом переходить от одной фазы движения к другой и наоборот, причем длительность пассивной фазы имеет экспоненциальное распределение, а длительность активной фазы (длина свободного пробега частицы) распределена по произвольному закону. В рамках предложенной модели выводится система уравнений, которым удовлетворяют плотности активных и пассивных частиц.

Кроме того, в работе выводится асимптотическое приближение к рассматриваемой модели в предположении, что переходы между фазами движения происходят очень часто. Приводится пример, демонстрирующий, что неверное представление о распределении длины свободного пробега частиц может приводить к значительной ошибке.

\section{1. Модель}

Частицы перемещаются в $d$-мерном евклидовом пространстве $\mathbf{R}^{d}$. Они могут находиться в одной из двух фаз: первая - баллистическая (активная) фаза, когда частица движется с постоянной скоростью в некотором направлении, вторая - броуновская (пассивная) фаза, когда частица находится в броуновском движении, при этом частица случайным образом переходит от одной фазы движения к другой, и наоборот (рис. 1). Предполагается, что длительность пассивной фазы имеет экспоненциальное распределение, а длительность активной фазы (длина свободного пробега частицы) распределена по произвольному закону.

Экспоненциальное распределение это единственное непрерывное распределение, которое обладает свойством „отсутствия последействия“ (точнее, „отсутствия памяти“, memoryless) $[14,15]$. Напомним, что случайная величина $X$ обладает свойством „отсутствия последействия“ (memoryless), если выполняется равенство

$$
P\left\{X>\tau^{\prime}+\tau \mid X>\tau^{\prime}\right\}=P\{X>\tau\},
$$

где $\tau, \tau^{\prime}$ - произвольные. Иными словами (в рассматриваемой интерпретации), каким бы ни было время $\tau^{\prime}$, в течение которого частица движется прямолинейно, распределение времени $\tau$, оставшегося до перехода к броуновскому движению, не зависит от $\tau^{\prime}$, и оно такое же, как если бы частица только начинала прямолинейное движение. 


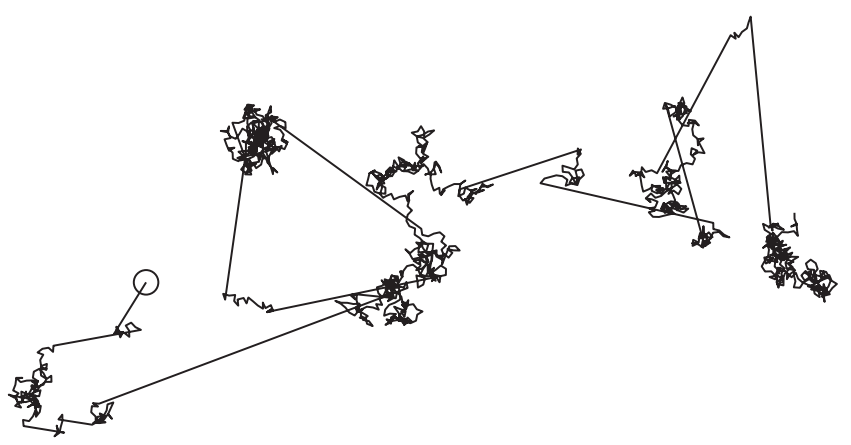

Рис. 1. Пример перемещения частицы (начало траектории отмечено кружком).

Таким образом, в случае неэкспоненциального распределения продолжительности активной фазы плотность активных частиц не может зависеть только от переменных $\mathbf{r}, t$ и $\mathbf{v}$ (координата, время и скорость соответственно). Необходимо введение дополнительной переменной $\tau$ - времени, прошедшего с начала текущей активной фазы. Назовем $\tau$ возрастом активной частицы (каждый раз, когда частица начинает прямолинейное движение, она рождается заново).

Плотности частиц в активной и пассивной фазах обозначим через $\xi(\mathbf{r}, t, \mathbf{v}, \tau)$ и $\vartheta(\mathbf{r}, t)$ соответственно, где $\mathbf{r}-$ координата в пространстве, $t-$ время, $\mathbf{v}=\left(v_{1}, \ldots, v_{d}\right) \in \mathbf{V}$ - вектор скорости активной частицы, $\tau$ - возраст активной частицы. Важно отметить, что в одной и той же точке пространства частица может находиться как в активном, так и в пассивном движении.

Плотность $\xi$ удовлетворяет уравнению, см. [16,17],

$$
\partial_{t} \xi+\partial_{\tau} \xi+\mathbf{v} \cdot \nabla \xi+\gamma \xi=0,
$$

где $\gamma(\mathbf{r}, \mathbf{v}, \tau)$ - интенсивность перехода (т.е. вероятность перехода в единицу времени) от активной фазы к пассивной, она зависит от возраста частицы, поскольку длительность активной фазы имеет произвольное распределение. Уравнение (1) описывает плотность невзаимодействующих частиц в точке $\mathbf{r}$, движущихся со скоростью $\mathbf{v}$, которые прекращают прямолинейное движение и начинают броуновское с интенсивностью $\gamma$, которая зависит от времени, в течение которого частицы двигались прямолинейно.

Уравнение (1) дополняется условием, задающим плотность вновь рожденных активных частиц (в точке пространства $\mathbf{r}$ в момент времени $t$ со скоростью $\mathbf{v})$,

$$
\left.\xi\right|_{\tau=0}=\alpha(\mathbf{r}) T(\mathbf{r}, \mathbf{v}) \vartheta
$$

где $\alpha-$ интенсивность перехода от пассивной к активной фазе, $T$ - ядро перехода от пассивной к активной фазе, удовлетворяющее условиям

$$
T \geq 0 \quad \text { и } \quad \int_{\mathbf{V}} T(\mathbf{r}, \mathbf{v}) d \mathbf{v}=1,
$$

т.е. $T$ как функция $\mathbf{v}-$ плотность распределения вероятностей. Отметим, что интенсивность перехода от пассивной к активной фазе не зависит от возраста броуновской частицы (т. е. времени, в течение которого частица находилась в текущей фазе броуновского движения), поскольку длительность пассивной фазы имеет экспоненциальное распределение.

Плотность $\vartheta$ удовлетворяет уравнению диффузии

$$
\partial_{t} \vartheta-D \Delta \vartheta=-\alpha \vartheta \int_{\mathbf{V}} \int_{0}^{t} \gamma(\mathbf{r}, \mathbf{v}, \tau) \xi(\mathbf{r}, t, \mathbf{v}, \tau) d \tau d \mathbf{v}
$$

где $D-$ коэффициент диффузии. Первое слагаемое в правой части выражает потерю броуновских частиц из-за перехода в активную фазу, второе слагаемое - источник броуновских частиц вследствие перехода из активной фазы.

Начальные условия имеют вид

$$
\left.\xi\right|_{t=0}=\chi(\mathbf{r}, \mathbf{v}) \delta(\tau),
$$

где $\delta(\cdot)$ - дельта-функция Дирака, и

$$
\left.\vartheta\right|_{t=0}=\vartheta_{0}(\mathbf{r})
$$

Начальное условие (5) означает, что в начальный момент $t=0$ все активные частицы находились в начале фазы, т. е. имели возраст, равный нулю.

\section{2. Преобразованные уравнения}

Избавимся от переменной $\tau$ в предложенной модели. Для этого определим плотность частиц в активной фазе

$$
\psi(\mathbf{r}, t, \mathbf{v})=\int_{0}^{t} \xi(\mathbf{r}, t, \mathbf{v}, \tau) d \tau
$$

не зависящую от их возраста (в момент времени $t$ не может быть активных частиц возраста $\tau>t$, см. начальное условие (5)).

Исключая возраст $\tau$ из уравнений $(1),(4)$ и действуя так же, как в [17], получим, что плотности $\psi$, $\vartheta$ удовлетворяют системе уравнений

$$
\begin{aligned}
& \partial_{t} \psi+\mathbf{v} \cdot \nabla \psi=-\hat{M} \psi(\mathbf{r}, t, \mathbf{v})+\alpha T \vartheta \\
& \partial_{t} \vartheta-D \Delta \vartheta=\int_{\mathbf{V}} \hat{M} \psi(\mathbf{r}, t, \mathbf{v}) d \mathbf{v}-\alpha \vartheta
\end{aligned}
$$

где

$$
\hat{M} \psi(\mathbf{r}, t, \mathbf{v})=\int_{0}^{t} \phi(\mathbf{r}, \mathbf{v}, \tau) \psi(\mathbf{r}-\mathbf{v} \tau, t-\tau, \mathbf{v}) d \tau
$$

- вообще говоря, нелокальный во времени оператор (оператор памяти, memory operator), $\phi$ - ядро памяти, 
определенное через свое преобразование Лапласа по формуле

$$
\begin{gathered}
\hat{L} \phi(\mathbf{r}, \mathbf{v}, s) \stackrel{\text { def }}{=} \frac{\hat{L} p(\mathbf{r}, \mathbf{v}, s)}{\hat{L} S(\mathbf{r}, \mathbf{v}, s)}, \\
\hat{L} f(s)=\int_{0}^{\infty} e^{-s \tau} f(\tau) d \tau
\end{gathered}
$$

- преобразование Лапласа,

$$
S(\mathbf{r}, \mathbf{v}, \tau)=\exp \left\{-\int_{0}^{\tau} \gamma\left(\mathbf{r}-\mathbf{v}\left(\tau-\tau^{\prime}\right), \mathbf{v}, \tau^{\prime}\right) d \tau^{\prime}\right\}
$$

- вероятность того, что частица, двигающаяся в точке $\mathbf{r}$ прямолинейно со скоростью $\mathbf{v}$, имеет возраст не менее $\tau$ (вероятность дожития, survival probability),

$$
p(\mathbf{r}, \mathbf{v}, \tau)=\gamma(\mathbf{r}, \mathbf{v}, \tau) S(\mathbf{r}, \mathbf{v}, \tau),
$$

$p(\mathbf{r}, \mathbf{v}, \tau) d \tau-$ вероятность того, что частица, движущаяся в точке $\mathbf{r}$ прямолинейно со скоростью $\mathbf{v}$ и имеющая возраст $\tau$, начнет броуновское движение в возрастном интервале $(\tau, \tau+d \tau)$. (Если $\gamma=$ const, то $p$ как функция $\tau$ является плотностью распределения вероятностей, но в общем случае это не так.)

Начальное условие для плотности $\psi$, следующее из начального условия (5) для плотности $\xi$, имеет вид

$$
\left.\psi\right|_{t=0}=\chi(\mathbf{r}, \mathbf{v}) .
$$

Заметим, что, если $\gamma=$ const (интенсивность перехода от активной фазы к пассивной не зависит от возраста частицы), то $\phi(\tau)=\gamma \delta(\tau)$ (т.е. частицы в активной фазе обладают свойством „отсутствия последействия“ (memoryless), и распределение времени жизни активной частицы экспоненциально: $\left.p(\tau)=\gamma e^{-\gamma \tau}\right)$ и, следовательно, $\hat{M} \psi=\gamma \psi$, т.е. оператор $\hat{M}$ становится локальным во времени. В этом случае уравнения (7), (8) аналогичны уравнениям в [18] при $d=2$.

\section{3. Асимптотическое приближение}

Нахождение аналитического решения задачи Коши для системы уравнений (7), (8) возможно лишь в исключительных случаях. Поэтому представляет интерес нахождение приближенных решений. Здесь находится асимптотическое приближение к рассматриваемой модели в предположении, что переходы между фазами движения происходят очень часто, т. е. средняя продолжительность жизни активных и пассивных частиц мала.

\section{1. Предположения}

Перечислим все предположения, которые используются при выводе асимптотического приближения.

Предполагается, что интенсивность перехода $\gamma$ не зависит от направления движения частицы $\Omega=\mathbf{v} / v$, $v=|\mathbf{v}|$, а зависит только от абсолютной величины скорости $v$. Следовательно, от направления $\Omega$ не зависят также вероятность дожития $S$, плотность $p$ и ядро памяти $\phi$.

Средняя продолжительность жизни активной частицы (т.е. средний возраст активной частицы, в котором она превращается в броуновскую)

$$
\left\langle\tau_{a}\right\rangle \equiv\left\langle\tau_{a}\right\rangle(\mathbf{r}, v)=\int_{0}^{\infty} \tau p(\mathbf{r}, v, \tau) d \tau
$$

и второй момент распределения возраста активной частицы

$$
\left\langle\tau_{a}^{2}\right\rangle \equiv\left\langle\tau_{a}^{2}\right\rangle(\mathbf{r}, v)=\int_{0}^{\infty} \tau^{2} p(\mathbf{r}, v, \tau) d \tau
$$

конечны.

Средняя продолжительность жизни активной частицы $\left\langle\tau_{a}\right\rangle$ является малой величиной порядка $\varepsilon$, где $\varepsilon-$ малый параметр. В этом случае интенсивность перехода $\gamma$ можно представить в виде

$$
\gamma(\mathbf{r}, v, \tau)=\frac{1}{\varepsilon} \bar{\gamma}\left(\mathbf{r}, v, \frac{\tau}{\varepsilon}\right),
$$

где $\bar{\gamma}$ не зависит от $\varepsilon$. Отсюда следуют представления

$$
\begin{gathered}
S(\mathbf{r}, v, \tau)=\bar{S}\left(\mathbf{r}, v, \frac{\tau}{\varepsilon}\right), p(\mathbf{r}, v, \tau)=\frac{1}{\varepsilon} \bar{p}\left(\mathbf{r}, v, \frac{\tau}{\varepsilon}\right), \\
\phi(\mathbf{r}, v, \tau)=\frac{1}{\varepsilon^{2}} \bar{\phi}\left(\mathbf{r}, v, \frac{\tau}{\varepsilon}\right),
\end{gathered}
$$

где $\bar{S}, \bar{p}, \bar{\phi}$ не зависят от $\varepsilon$.

Средняя продолжительность жизни пассивной частицы $\left\langle\tau_{d}\right\rangle$ (т.е. средний возраст броуновской частицы, в котором она превращается в активную) также является малой величиной порядка $\varepsilon$. Поскольку $\left\langle\tau_{d}\right\rangle=1 / \alpha$, это означает, что интенсивность перехода от пассивной к активной фазе имеет вид

$$
\alpha(\mathbf{r})=\frac{\bar{\alpha}(\mathbf{r})}{\varepsilon}
$$

где $\bar{\alpha}$ не зависит от $\varepsilon$.

Коэффициент диффузии $D$ является малой величиной порядка $\varepsilon$, т. е.

$$
D=\varepsilon \bar{D},
$$

где $\bar{D}$ не зависит от $\varepsilon$.

Ядро перехода от пассивной к активной фазе имеет вид

$$
T(\mathbf{r}, \mathbf{v})=\bar{T}(\mathbf{r}, \mathbf{v})+\varepsilon \tilde{T}(\mathbf{r}, \mathbf{v}),
$$

где $\bar{T}$ и $\tilde{T}$ не зависят от $\varepsilon$, причем

$$
\bar{T} \geq 0 \quad \text { и } \int_{\mathbf{V}} \bar{T}(\mathbf{r}, \mathbf{v}) d \mathbf{v}=1 .
$$


Предполагается также, что для невозмущенной части ядра справедливо соотношение

$$
\bar{T}(\mathbf{r},-\mathbf{v})=\bar{T}(\mathbf{r}, \mathbf{v}) .
$$

Это условие выполняется, например, если $\bar{T}$ изотропно, т.е. $\bar{T}=|\mathbf{V}|^{-1}$, где $|\mathbf{V}|$ - объем $\mathbf{V}$. Из условий (3), (10) следует, что

$$
\int_{\mathbf{V}} \tilde{T}(\mathbf{r}, \mathbf{v}) d \mathbf{v}=0
$$

Пространство скоростей $\mathbf{V}$ ограничено и инвариантно относительно вращений вокруг начала координат, и не содержит нулевую скорость.

\section{2. Асимптотическое разложение}

С учетом сделанных предположений уравнения (7), (8) принимают вид

$$
\begin{gathered}
\partial_{t} \psi+\mathbf{v} \cdot \nabla \psi=-\frac{1}{\varepsilon} \hat{M}_{\varepsilon} \psi(\mathbf{r}, t, \mathbf{v})+\frac{\bar{\alpha}}{\varepsilon}(\bar{T}+\varepsilon \tilde{T}) \vartheta \\
\partial_{t} \vartheta-\varepsilon \bar{D} \Delta \vartheta=\frac{1}{\varepsilon} \int_{\mathbf{V}} \hat{M}_{\varepsilon} \psi(\mathbf{r}, t, \mathbf{v}) d \mathbf{v}-\frac{\bar{\alpha}}{\varepsilon} \vartheta
\end{gathered}
$$

с оператором памяти

$$
\hat{M}_{\varepsilon} \psi(\mathbf{r}, t, \mathbf{v})=\int_{0}^{t} \frac{1}{\varepsilon} \bar{\phi}\left(\mathbf{r}, v, \frac{\tau}{\varepsilon}\right) \psi(\mathbf{r}-\mathbf{v} \tau, t-\tau, \mathbf{v}) d \tau .
$$

Представим плотности в виде

$$
\begin{gathered}
\psi(\mathbf{r}, t, \mathbf{v})=\psi^{\circ}\left(\mathbf{r}, t^{\circ}, \mathbf{v}\right)+\psi^{i}\left(\mathbf{r}, t^{i}, \mathbf{v}\right), \\
\vartheta(\mathbf{v}, t)=\vartheta^{\circ}\left(\mathbf{r}, t^{\circ}\right)+\vartheta^{i}\left(\mathbf{r}, t^{i}\right),
\end{gathered}
$$

где

$$
t^{\circ}=\varepsilon t \quad \text { и } \quad t^{i}=\frac{t}{\varepsilon}
$$

- медленное и быстрое время соответственно, $\psi^{\circ}$, $\vartheta^{\circ}$ и $\phi^{i}, \vartheta^{i}-$ внешние и внутренние решения соответственно. Внутренние решения аппроксимируют $\psi$ и $\vartheta$ в пограничном слое в окрестности $t=0$, т.е. при $0<t \lesssim \mathrm{O}(\varepsilon)$, внешние решения аппроксимируют $\psi$ и $\vartheta$ вне этого пограничного слоя, т.е. при $t \gtrsim \mathrm{O}(\varepsilon)$. Здесь будет выведена асимптотика только для внешних решений. Асимптотика для внутренних ре шений может быть выведена так же, как в [17].

Подставляя представления (15),(16) в уравнения (12), (13), получим, что внешние решения удовлетворяют уравнениям

$$
\begin{aligned}
\varepsilon^{2} \partial_{t^{\circ}} \psi^{\circ}+\varepsilon \mathbf{v} \cdot \nabla \psi^{\circ} & =-\hat{M}_{\varepsilon} \psi^{\circ}\left(\mathbf{r}, t^{\circ}, \mathbf{v}\right)+\bar{\alpha}(\bar{T}+\varepsilon \tilde{T}) \vartheta^{\circ} \\
\varepsilon^{2} \partial_{t^{\circ}} \vartheta^{\circ}+\varepsilon^{2} \bar{D} \Delta \vartheta^{\circ} & =\int_{\mathbf{v}} \hat{M}_{\varepsilon} \psi^{\circ}\left(\mathbf{r}, t^{\circ}, \mathbf{v}\right) d \mathbf{v}-\bar{\alpha} \vartheta^{\circ}
\end{aligned}
$$

Ядро памяти представляется асимптотическим разложением [17]

$$
\frac{1}{\varepsilon} \bar{\phi}\left(\mathbf{r}, v, \frac{\tau}{\varepsilon}\right) \sim \bar{\phi}_{0} \delta(\tau)+\bar{\phi}_{1} \delta^{\prime}(\tau) \varepsilon+\bar{\phi}_{2} \delta^{\prime \prime}(\tau) \varepsilon^{2}+\ldots
$$

$$
\text { при } \varepsilon \rightarrow 0
$$

(разложение понимается в слабом смысле) с коэффициентами

$$
\bar{\phi}_{0} \equiv \bar{\phi}_{0}(\mathbf{r}, v)=\frac{1}{\left\langle\bar{\tau}_{a}\right\rangle}, \quad \bar{\phi}_{1} \equiv \bar{\phi}_{1}(\mathbf{r}, v)=\frac{\left\langle\bar{\tau}_{a}^{2}\right\rangle}{2\left\langle\bar{\tau}_{a}\right\rangle^{2}}-1,
$$

где

$$
\left\langle\bar{\tau}_{a}\right\rangle \equiv\left\langle\bar{\tau}_{a}\right\rangle(\mathbf{r}, v) \int_{0}^{\infty} \tau \bar{p}(\mathbf{r}, v, \tau) d \tau
$$

и

$$
\left\langle\bar{\tau}_{a}^{2}\right\rangle \equiv\left\langle\bar{\tau}_{a}^{2}\right\rangle(\mathbf{r}, v)=\int_{0}^{\infty} \tau^{2} \bar{p}(\mathbf{r}, v, \tau) d \tau
$$

- первый (среднее) и второй моменты распределения $\bar{p}$ (эти коэффициенты и моменты не зависят от $\varepsilon$ ).

Подставляя асимптотическое разложение ядра памяти (19) в выражение для оператора памяти (14), получим асимптотическое разложение

$$
\begin{aligned}
& \hat{M}_{\varepsilon} \psi^{\circ}\left(\mathbf{r}, t^{\circ}, \mathbf{v}\right)=\int_{0}^{t^{\circ} / \varepsilon} \frac{1}{\varepsilon} \bar{\phi}\left(\mathbf{r}, v, \frac{\tau}{\varepsilon}\right) \phi^{\circ}\left(\mathbf{r}-\mathbf{v} \tau, t^{\circ}-\varepsilon \tau, \mathbf{v}\right) d \tau \\
& \left.\equiv\left\{\bar{\phi}_{0}+\varepsilon \bar{\phi}_{1}(\mathbf{v} \cdot \nabla)+\varepsilon^{2}\left[\bar{\phi}_{1} \partial_{t^{\circ}}+\bar{\phi}_{2}(\mathbf{v} \cdot \nabla)^{2}\right]+\ldots\right]\right\} \psi^{\circ} .
\end{aligned}
$$

Запишем уравнения (17),(18), учитывая разложение $(20)$, в матрично-операторном виде

$$
\left(\varepsilon^{2} \hat{D}+\varepsilon \hat{V}\right)\left(\begin{array}{l}
\psi^{\circ} \\
\vartheta^{\circ}
\end{array}\right)=\left[-(\hat{I}-\hat{T}) \hat{B}_{0}+\varepsilon \hat{A}_{1}+\varepsilon^{2} \hat{A}_{2}+\ldots\right]\left(\begin{array}{l}
\psi^{\circ} \\
\vartheta^{\circ}
\end{array}\right),
$$

где $\hat{I}$ - тождественная матрица-оператор, матрицаоператор $\hat{T}$ имеет вид

$$
\hat{T}=\left(\begin{array}{cc}
0 & \bar{T}(\mathbf{r}, \mathbf{v}) \\
\int_{\mathbf{v}} d \mathbf{v} & 0
\end{array}\right)
$$

она действует следующим образом:

$$
\hat{T}\left(\begin{array}{c}
\psi \\
\vartheta
\end{array}\right)=\left(\begin{array}{c}
\bar{T}(\mathbf{r}, \mathbf{v}) \vartheta(\mathbf{r}, t) \\
\int_{\mathbf{v}} \psi(\mathbf{r}, t, \mathbf{v}) d \mathbf{v}
\end{array}\right),
$$

остальные матрицы-операторы имеют вид

$$
\hat{D}=\left(\begin{array}{cc}
\partial_{t^{\circ}} & 0 \\
0 & \partial_{t^{\circ}}-\bar{D} \Delta
\end{array}\right), \hat{V}=\left(\begin{array}{cc}
\mathbf{v} \cdot \nabla & 0 \\
0 & 0
\end{array}\right),
$$




$$
\begin{gathered}
\hat{B}_{0}=\left(\begin{array}{cc}
\bar{\phi}_{0} & 0 \\
0 & \bar{\alpha}
\end{array}\right) \equiv\left(\begin{array}{cc}
1 /\left\langle\bar{\tau}_{a}\right\rangle & 0 \\
0 & 1 /\left\langle\bar{\tau}_{d}\right\rangle
\end{array}\right), \\
\hat{A}_{1}=-(\hat{I}-\hat{T})\left(\begin{array}{cc}
\bar{\phi}_{1}(\mathbf{v} \cdot \nabla) & 0 \\
0 & 0
\end{array}\right)+\left(\begin{array}{cc}
0 & \bar{\alpha} \tilde{T} \\
0 & 0
\end{array}\right), \\
\hat{A}_{2}=-(\hat{I}-\hat{T})\left(\begin{array}{cc}
\bar{\phi}_{1} \partial_{t^{\circ}}+\bar{\phi}_{2}(\mathbf{v} \cdot \nabla)^{2} & 0 \\
0 & 0
\end{array}\right) .
\end{gathered}
$$

Мы предполагаем, что внешние решения имеют асимптотическое разложение

$$
\left(\begin{array}{c}
\psi^{\circ} \\
\vartheta^{\circ}
\end{array}\right) \sim\left(\begin{array}{l}
\psi_{0}^{\circ} \\
\vartheta_{0}^{\circ}
\end{array}\right)+\left(\begin{array}{c}
\psi_{1}^{\circ} \\
\vartheta_{1}^{\circ}
\end{array}\right) \varepsilon+\left(\begin{array}{c}
\psi_{2}^{\circ} \\
\vartheta_{2}^{\circ}
\end{array}\right) \varepsilon^{2}+\ldots \text { при } \varepsilon \rightarrow 0 .
$$

Подставляя это асимптотическое разложение в уравнение (21) и приравнивая коэффициенты при одинаковых степенях $\varepsilon$, получим следующие уравнения:

$$
\begin{gathered}
\varepsilon^{0}:(\hat{I}-\hat{T}) \hat{B}_{0}\left(\begin{array}{c}
\psi_{0}^{\circ} \\
\vartheta_{0}^{\circ}
\end{array}\right)=\left(\begin{array}{l}
0 \\
0
\end{array}\right), \\
\varepsilon^{1}:-(\hat{I}-\hat{T}) \hat{B}_{0}\left(\begin{array}{c}
\psi_{1}^{\circ} \\
\vartheta_{1}^{\circ}
\end{array}\right)=\left(\hat{V}-\hat{A}_{1}\right)\left(\begin{array}{l}
\psi_{0}^{\circ} \\
\vartheta_{0}^{\circ}
\end{array}\right), \\
\varepsilon^{2}:-(\hat{I}-\hat{T}) \hat{B}_{0}\left(\begin{array}{c}
\psi_{2}^{\circ} \\
\vartheta_{2}^{\circ}
\end{array}\right)=\left(\hat{D}-\hat{A}_{2}\right)\left(\begin{array}{c}
\psi_{0}^{\circ} \\
\vartheta_{0}^{\circ}
\end{array}\right)+\left(\hat{V}-\hat{A}_{1}\right)\left(\begin{array}{c}
\psi_{1}^{\circ} \\
\vartheta_{1}^{\circ}
\end{array}\right),
\end{gathered}
$$

Рассмотрим уравнение (23a). Согласно теоремам Фредгольма, уравнение

$$
(\hat{I}-\hat{T})\left(\begin{array}{l}
\psi \\
\vartheta
\end{array}\right)=\left(\begin{array}{l}
0 \\
0
\end{array}\right)
$$

и уравнение

$$
\left(\hat{I}-\hat{T}^{*}\right)\left(\begin{array}{l}
\psi \\
\vartheta
\end{array}\right)=\left(\begin{array}{l}
0 \\
0
\end{array}\right)
$$

имеют одинаковое (с точностью до множителя, не зависящего от $\mathbf{v}$ ) число нетривиальных решений. Заметим, что сопряженная матрица-оператор $\hat{T}^{*}$ имеет вид

$$
\hat{T}^{*}=\left(\begin{array}{cc}
0 & 1 \\
\int_{\mathbf{v}} d \mathbf{v} \bar{T}(\mathbf{r}, \mathbf{v}) & 0
\end{array}\right),
$$

она действует следующим образом:

$$
\hat{T}^{*}\left(\begin{array}{l}
\psi \\
\vartheta
\end{array}\right)=\left(\begin{array}{c}
\vartheta(\mathbf{r}, t) \\
\int_{\mathbf{v}} \bar{T}(\mathbf{r}, \mathbf{v}) \psi(\mathbf{r}, t, \mathbf{v}) d \mathbf{v}
\end{array}\right) .
$$

Нетрудно видеть, что вектор $\left(\begin{array}{ll}1 & 1\end{array}\right)^{T}$ - единственное (с точностью до множителя, не зависящего от $\mathbf{v}$ ) решение уравнения (25). Вектор $(\bar{T}(\mathbf{r}, \mathbf{v}) 1)^{T}$ является решением уравнения (24). Следовательно, решение уравнения (23a) имеет вид

$$
\left(\begin{array}{c}
\psi_{0}^{\circ} \\
\vartheta_{0}^{\circ}
\end{array}\right)=\hat{B}_{0}^{-1}\left(\begin{array}{c}
\bar{T}(\mathbf{r}, \mathbf{v}) \\
1
\end{array}\right) \frac{\rho}{\bar{C}} \equiv\left(\begin{array}{c}
\left\langle\bar{\tau}_{a}\right\rangle \bar{T}(\mathbf{r}, \mathbf{v}) \\
\left\langle\bar{\tau}_{d}\right\rangle
\end{array}\right) \frac{\rho}{\bar{C}},
$$

где $\left\langle\bar{\tau}_{d}\right\rangle=1 / \bar{\alpha}, \rho \equiv \rho\left(\mathbf{r}, t^{\circ}\right)-$ функция (независимый от $\mathbf{v}$ множитель), требующая определения,

$$
\bar{C} \equiv \bar{C}(\mathbf{r})=\int_{\mathbf{v}}\left\langle\bar{\tau}_{a}\right\rangle \bar{T}(\mathbf{r}, \mathbf{v}) d \mathbf{v}+\left\langle\bar{\tau}_{d}\right\rangle
$$

- нормирующая функция, введенная для того, чтобы выполнялось соотношение

$$
\rho\left(\mathbf{r}, t^{\circ}\right)=\int_{\mathbf{V}} \psi_{0}^{\circ}\left(\mathbf{r}, t^{\circ}, \mathbf{v}\right) d \mathbf{v}+\vartheta_{0}^{\circ}\left(\mathbf{r}, t^{\circ}\right),
$$

т. е. $\rho$ - суммарная плотность всех частиц в точке $\mathbf{r}$ при $\varepsilon \rightarrow 0$.

Рассмотрим уравнение (23b). Согласно теоремам Фредгольма, необходимым и досточным условием разрешимости этого уравнения является ортогональность правой части вектору $\left(\begin{array}{ll}1 & 1\end{array}\right)^{T}$ (нетривиальному решению уравнения (25)). Применяя условие разрешимости, получим эквивалентное условие

$$
\begin{aligned}
& \int_{\mathbf{v}} \mathbf{v} \cdot \nabla\left[\left\langle\bar{\tau}_{a}\right\rangle \bar{T}(\mathbf{r}, \mathbf{v}) \frac{\rho}{\bar{C}}\right] d \mathbf{v} \\
& \equiv \nabla \cdot\left\{\left[\int_{\mathbf{v}} \mathbf{v}\left\langle\bar{\tau}_{a}\right\rangle \bar{T}(\mathbf{r}, \mathbf{v}) d \mathbf{v}\right] \frac{\rho}{\bar{C}}\right\}=0 .
\end{aligned}
$$

Если выполняется условие (11), то

$$
\int_{\mathbf{V}} \mathbf{v}\left\langle\bar{\tau}_{a}\right\rangle \bar{T}(\mathbf{r}, \mathbf{v}) d \mathbf{v}=0,
$$

и, следовательно, условие разрешимости выполнено. В этом случае решение уравнения (23b) имеет вид

$$
\left(\begin{array}{c}
\psi_{1}^{\circ} \\
\vartheta_{1}^{\circ}
\end{array}\right)=-\hat{B}_{0}^{-1}\left\{\left(\begin{array}{c}
\left(1+\bar{\phi}_{1}\right)(\mathbf{v} \cdot \nabla) \bar{T} / \bar{\phi}_{0}-\tilde{T} \\
0
\end{array}\right) \frac{\rho}{\bar{C}}+\left(\begin{array}{l}
\bar{T} \\
1
\end{array}\right) c\right\},
$$

где $c \equiv c\left(\mathbf{r}, t^{\circ}\right)-$ произвольная функция.

Рассмотрим уравнение (23c). Записывая условие разрешимости к этому уравнению (ортогональность правой части вектору $\left.\left(\begin{array}{ll}1 & 1\end{array}\right)^{T}\right)$ и подставляя в него решения (26), (28), получим, что плотность $\rho$ удовлетворяет уравнению анизотропной диффузии со сносом

$$
\begin{aligned}
\partial_{t} \circ \rho & -\bar{D} \Delta\left[\left\langle\bar{\tau}_{d}\right\rangle \frac{\rho}{\bar{C}}\right]-\nabla \cdot\left\{\int_{\mathbf{V}} \frac{\left\langle\bar{\tau}_{a}^{2}\right\rangle}{2\left\langle\bar{\tau}_{a}\right\rangle} \mathbf{v v}^{T} \nabla\left[\left\langle\bar{\tau}_{a}\right\rangle \bar{T} \frac{\rho}{\bar{C}}\right] d \mathbf{v}\right\} \\
& +\nabla \cdot\left\{\left[\int_{\mathbf{v}}\left\langle\bar{\tau}_{a}\right\rangle \tilde{T} \mathbf{v} d \mathbf{v}\right] \frac{\rho}{\bar{C}}\right\}=0 .
\end{aligned}
$$

Это уравнение записано относительно медленного времени $t^{\circ}$.

Умножая это уравнение на $\varepsilon$, получим, что в исходных переменных плотность $\rho$ удовлетворяет уравнению 
анизотропной диффузии со сносом

$$
\begin{aligned}
\partial_{t} \rho & -D \Delta\left[\left\langle\tau_{d}\right\rangle \frac{\rho}{C}\right]-\nabla \cdot\left\{\int_{\mathbf{V}} \frac{\left\langle\tau_{a}^{2}\right\rangle}{2\left\langle\tau_{a}\right\rangle} \mathbf{v v}^{T} \nabla\left[\left\langle\tau_{a}\right\rangle \bar{T} \frac{\rho}{C}\right] d \mathbf{v}\right\} \\
+ & \nabla \cdot\left\{\left[\int_{\mathbf{V}}\left\langle\tau_{a}\right\rangle(T-\bar{T}) \mathbf{v} d \mathbf{v}\right] \frac{\rho}{C}\right\}=0
\end{aligned}
$$

где нормирующая функция имеет вид

$$
C \equiv C(\mathbf{r})=\int_{\mathbf{V}}\left\langle\tau_{a}\right\rangle \bar{T}(\mathbf{r}, \mathbf{v}) d \mathbf{v}+\left\langle\tau_{d}\right\rangle .
$$

Уравнение (29) является именно уравнением диффузии со сносом. Это можно увидеть, если проделать очевидные преобразования, но тогда уравнение станет более громоздким.

Начальное условие для плотности $\rho$ следует из соотношения (27) и начальных условий (5), (6), оно имеет вид

$$
\left.\rho\right|_{t=0}=\int_{\mathbf{V}} \chi(\mathbf{r}, \mathbf{v}) d \mathbf{v}+\vartheta_{0}(\mathbf{r}) .
$$

Плотность $\rho$ - суммарная плотность всех частиц в точке $\mathbf{r}$ при $\varepsilon \rightarrow 0$. Плотности активных и пассивных частиц находятся из плотности $\rho$ по формулам

$$
\begin{gathered}
\psi_{0}^{\circ}(\mathbf{r}, t, \mathbf{v})=\frac{\left\langle\tau_{a}\right\rangle \bar{T}(\mathbf{r}, \mathbf{v})}{C(\mathbf{r})} \rho(\mathbf{r}, t), \\
\vartheta_{0}^{\circ}(\mathbf{r}, t)=\frac{\left\langle\tau_{d}\right\rangle}{C(\mathbf{r})} \rho(\mathbf{r}, t)
\end{gathered}
$$

(эти формулы получаются из (26) приведением к исходному времени $t$ ).

\section{3. Частные случаи}

Рассмотрим частные случаи уравнения (29).

Предположим, что интенсивности перехода $\alpha$ и $\gamma$ не зависят от координаты $\mathbf{r}$ (но $\gamma$ зависит от $v=|\mathbf{v}|)$. В этом случае уравнение диффузии (29) принимает вид уравнения Фоккера-Планка

$$
\partial_{t} \rho-\sum_{i, j=1}^{d} \partial_{x_{i}} \partial_{x_{j}}\left[D_{i j}^{\mathrm{eff}}(\mathbf{r}) \rho\right]+\sum_{i=1}^{d} \partial_{x_{i}}\left[u_{i}(\mathbf{r}) \rho\right]=0,
$$

где $D_{i j}^{\mathrm{eff}}$ - элементы эффективного тензора диффузии

$$
\mathbf{D}^{\mathrm{eff}}(\mathbf{r})=\frac{1}{C(\mathbf{r})}\left[D\left\langle\tau_{d}\right\rangle \mathbf{I}+\int_{\mathbf{V}} \frac{\left\langle\tau_{a}^{2}\right\rangle}{2} \mathbf{v v}^{T} \bar{T}(\mathbf{r}, \mathbf{v}) d \mathbf{v}\right]
$$

$I$ - единичная матрица, $u_{i}$ - координаты вектора сноса

$$
\mathbf{u}(\mathbf{r})=\frac{1}{C(\mathbf{r})} \int_{\mathbf{V}}\left\langle\tau_{a}\right\rangle[T(\mathbf{r}, \mathbf{v})-\bar{T}(\mathbf{r}, \mathbf{v})] \mathbf{v} d \mathbf{v}
$$

(здесь $\left\langle\tau_{a}\right\rangle$ и $\left\langle\tau_{a}^{2}\right\rangle$ зависят от $\left.v\right), C(\mathbf{r})$ определяется по формуле (30).

Если среда однородна, т. е. от $\mathbf{r}$ не зависят (помимо $\alpha$ и $\gamma$ ) также и $T, \bar{T}$ и $\tilde{T}$ (а, следовательно, и $C$ ), уравнение Фоккера-Планка (32) принимает вид уравнения анизотропной диффузии со сносом

$$
\partial_{t} \rho-\nabla \cdot\left(\mathbf{D}^{\mathrm{eff}} \nabla \rho\right)+\mathbf{u} \cdot \nabla \rho=0,
$$

где

$$
\mathbf{D}^{\mathrm{eff}}=\frac{1}{C}\left[D\left\langle\tau_{d}\right\rangle \mathbf{I}+\int_{\mathbf{v}} \frac{\left\langle\tau_{a}^{2}\right\rangle}{2} \mathbf{v v}^{T} \bar{T}(\mathbf{v}) d \mathbf{v}\right]
$$

— постоянный эффективный тензор диффузии,

$$
\mathbf{u}=\frac{1}{C} \int_{\mathbf{V}}\left\langle\tau_{a}\right\rangle[T(\mathbf{v})-\bar{T}(\mathbf{v})] \mathbf{v} d \mathbf{v}
$$

- постоянный вектор сноса.

Предположим, что в пассивной фазе частица покоится, т.е. $D=0$, и, следовательно, $\vartheta$ обозначает плотность покоящихся частиц. Предположим также, что интенсивность перехода $\gamma$ не зависит от времени жизни и скорости движения активной частицы, т.е. $\gamma=\gamma(\mathbf{r})$, и движение частиц в активной фазе изотропно, т.е. $T(\mathbf{r}, \mathbf{v})=\bar{T}(\mathbf{r}, \mathbf{v})=|\mathbf{V}|^{-1}(|\mathbf{V}|-$ объем $\mathbf{V})$. В этом случае $\left\langle\tau_{a}\right\rangle=1 / \gamma(\mathbf{r}),\left\langle\tau_{d}^{2}\right\rangle=2 / \gamma^{2}(\mathbf{r})$, $\left\langle\tau_{d}\right\rangle=1 / \alpha(\mathbf{r}), C(\mathbf{r})=1 / \gamma(\mathbf{r})+1 / \alpha(\mathbf{r})$ и уравнение диффузии (29) принимает вид

$$
\partial_{t} \rho-\nabla \cdot\left\{\frac{1}{\gamma(\mathbf{r})} \frac{1}{|\mathbf{V}|}\left[\int_{\mathbf{V}} \mathbf{v v}^{T} d \mathbf{v}\right] \nabla\left[\frac{\alpha(\mathbf{r})}{\gamma(\mathbf{r})+\alpha(\mathbf{r})} \rho\right]\right\}=0 .
$$

Аналогичное уравнение получается в частном случае в [19].

Предположим, что пассивная фаза отсутствует, т.е. $\left\langle\tau_{d}\right\rangle=0$. Предположим также, что $\gamma=$ const, т.е. pacпределение времени жизни активной частицы экспоненциально, причем оно не зависит от скорости движения частицы (это довольно сильное ограничение). В этом случае $\left\langle\tau_{a}\right\rangle=1 / \gamma,\left\langle\tau_{a}^{2}\right\rangle=2 / \gamma^{2}, C(\mathbf{r})=1 / \gamma$. Кроме того, предположим, что ядро перехода от пассивной к активной фазе имеет вид $T(\mathbf{r}, \mathbf{v})=\bar{T}(\mathbf{r}, \mathbf{v})$, т.е. $\tilde{T}(\mathbf{r}, \mathbf{v})=0$. В этом случае вектор сноса равен нулю: $\mathbf{u}=0$. В результате уравнение Фоккера-Планка (32) принимает вид

$$
\partial_{t} \rho-\sum_{i, j=1}^{d} \partial_{x_{i}} \partial_{x_{j}}\left[D_{i j}^{\mathrm{eff}}(\mathbf{r}) \rho\right]=0,
$$

где эффективный тензор диффузии равен

$$
\mathbf{D}^{\mathrm{eff}}(\mathbf{r})=\frac{1}{\gamma} \int_{\mathbf{V}} \bar{T}(\mathbf{r}, \mathbf{v}) \mathbf{v} \mathbf{v}^{T} d \mathbf{v} .
$$

Это совпадает с уравнением, выведенным в [20]. Плотность активных частиц при $\varepsilon \rightarrow 0$ равна

$$
\psi_{0}^{\circ}(\mathbf{r}, t, \mathbf{v})=\bar{T}(\mathbf{r}, \mathbf{v}) \rho(\mathbf{r}, t),
$$

cM. (31). 


\section{4. Пример}

Представление об экспоненциальном распределении длины свободного пробега частиц широко распространено. Если такое распределение действительно экспоненциально, то достаточно оценить среднюю длину свободного пробега (среднюю длительность активной фазы), все остальные моменты экспоненциального распределения выражаются через его среднее. Однако распределение длины свободного пробега экспоненциально далеко не всегда. Ошибка, вызванная неверным представлением об этом распределении, может быть значительной.

В качестве примера рассмотрим односкоростную модель, в которой пространство скоростей имеет вид $\mathbf{V}=v \mathbf{S}^{d-1}$, где $\mathbf{S}^{d-1}$ - единичная сфера в $\mathbf{R}^{d}, v=$ const. Предположим для простоты, что среда однородна, $\left\langle\tau_{d}\right\rangle=0$ (пассивная фаза отсутствует или пренебрежимо коротка) и $T(\mathbf{r}, \mathbf{v})=\bar{T}(\mathbf{r}, \mathbf{v})=|\mathbf{V}|^{-1}$ (движение частиц в активной фазе изотропно). Учитывая соотношение,

$$
\int_{\mathbf{S}^{d-1}} \Omega \Omega^{T} d \Omega=\frac{\left|\mathbf{S}^{d-1}\right|}{d} \mathbf{I},
$$

где $\left|\mathbf{S}^{d-1}\right|-$ площадь единичной сферы, $\mathbf{I}-$ единичная матрица, получим, что эффективный тензор диффузии равен

$$
\mathbf{D}^{\mathrm{eff}}=D^{\mathrm{eff}} \mathbf{I}, \quad D^{\mathrm{eff}}=\frac{\left\langle\tau_{a}^{2}\right\rangle v^{2}}{2 d\left\langle\tau_{a}\right\rangle},
$$

а вектор сноса равен нулю. В результате уравнение Фоккера-Планка (32) принимает вид уравнения диффузии

$$
\partial_{t} \rho-D^{\mathrm{eff}} \Delta \rho=0, \quad \mathbf{r} \in \mathbf{R}^{d} .
$$

Зададим начальное условие

$$
\left.\rho\right|_{r=0}=\delta(\mathbf{r})
$$

и сравним решения задачи (34), (35), вычисляя коффициент диффузии по формуле (33) для экспоненциального и неэкспоненциального распределений продолжительности активной фазы.

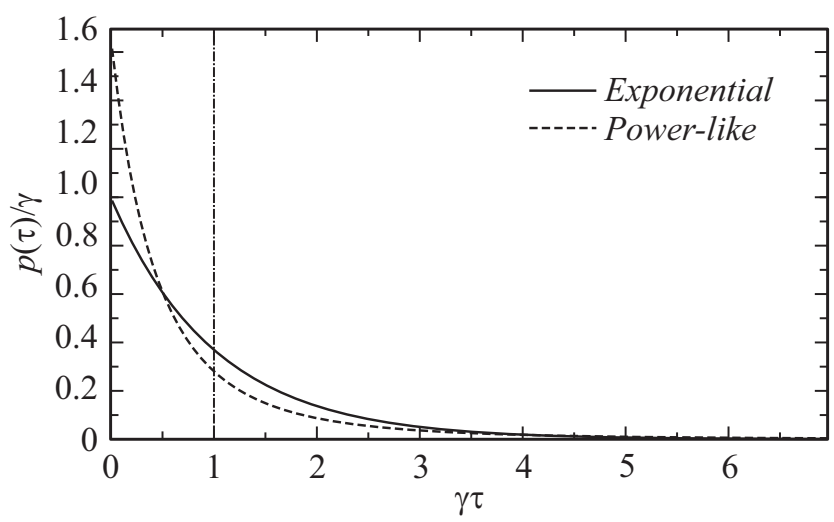

Рис. 2. Экспоненциальное и степенное $(v=2.5)$ распределения, имеющие одинаковое среднее (отмечено вертикальной штрихпунктирной линией).

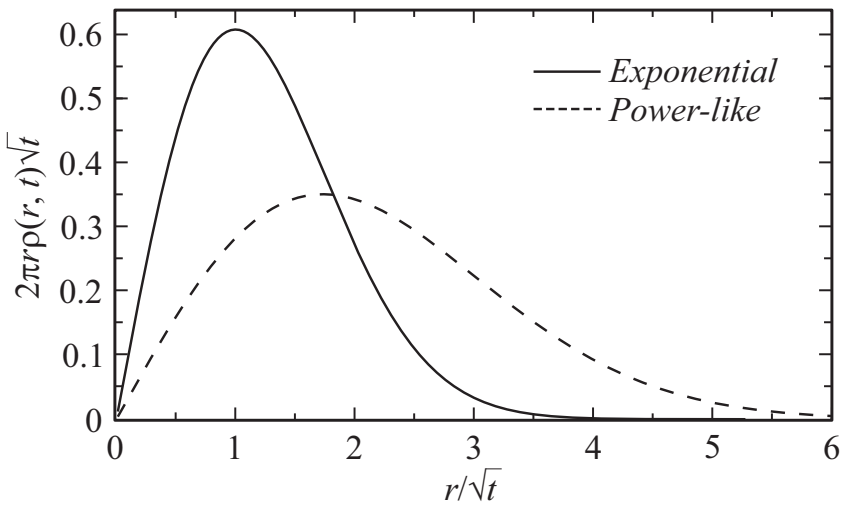

Рис. 3. Решения задачи (34), (35) с коэффициентом диффузии, соответствующим экспоненциальному и степенному $(v=2.5)$ распределениям, $d=2$. (В выбранном масштабе решения остаются неизменными.)

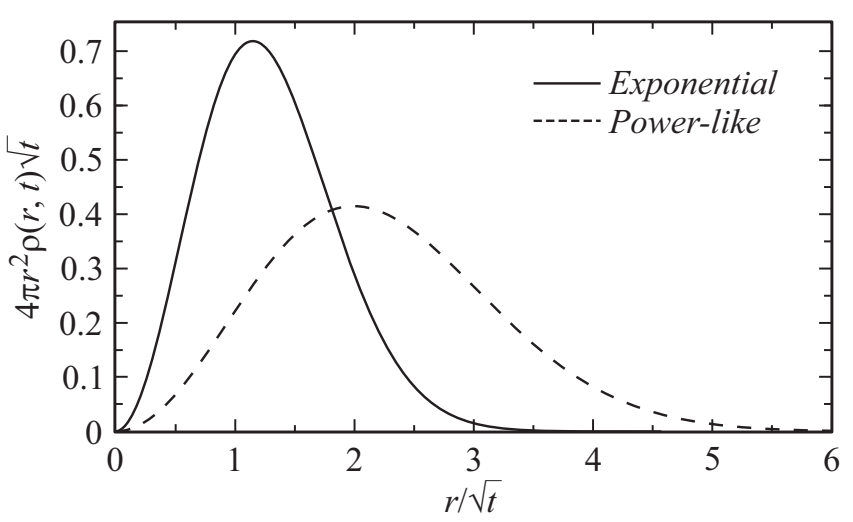

Рис. 4. Решения задачи (34), (35) с коэффициентом диффузии, соответствующим экспоненциальному и степенному $(v=2.5)$ распределениям, $d=3$. (В выбранном масштабе решения остаются неизменными.)

На рис. 2 показаны два распределения: экспоненциальное

$$
p(\tau)=\gamma e^{-\gamma \tau}
$$

и степенное

$$
p(\tau)=\frac{\gamma \nu}{v-1}\left[1+\frac{\gamma \tau}{v-1}\right]^{-(v+1)}
$$

$\left(p(\tau) \propto \tau^{-(v+1)}\right.$ при $\left.\tau \rightarrow \infty\right)$. Оба распределения имеют одинаковое среднее $\left\langle\tau_{a}\right\rangle=\gamma^{-1}$. Для экспоненциального распределения $\left\langle\tau_{a}^{2}\right\rangle=2 \gamma^{-2}$. Для степенного распределения $\left\langle\tau_{a}^{2}\right\rangle=2[(v-1) /(v-2)] \gamma^{-2}$. В результате получим, что эффективные коэффициенты диффузии равны соответственно

$$
D_{\mathrm{exp}}^{\mathrm{eff}}=\frac{v^{2}}{d \gamma} \text { и } D_{\mathrm{pow}}^{\mathrm{eff}}=\frac{v-1}{v-2} \frac{v^{2}}{d \gamma} .
$$

На рис. 3,4 показаны решения задачи (34), (35). На каждом из рисунков представлены решения с коэффициентом диффузии $D^{\mathrm{eff}}$, вычисленным по формуле (33) 
для каждого из двух указанных распределений, для степенного распределения $v=2.5$ (в обоих случаях $v=1)$. Коэффициент диффузии, соответствующий степенному распределению, в три раза больше коэффициента диффузии, соответствующего экспоненциальному распределению.

\section{Заключение}

В работе предложена модель переноса частиц (молекул или биологических особей), в котором чередуются баллистическое (прямолинейное) и броуновское движение, причем длительность броуновской фазы имеет экспоненциальное распределение, а длительность баллистической фазы (длина свободного пробега частиц) распределена по произвольному закону. В рамках модели выведена система уравнений, которым удовлетворяют плотности частиц в каждой из фаз.

Нахождение аналитических решений в рамках предложенной модели возможно лишь в исключительных случаях. Поэтому выведено асимптотическое приближение к этой модели в предположении, что переходы между фазами движения происходят очень часто. Это приближение описывается уравнением анизотропной диффузии со сносом. Рассмотрены частные случаи этого уравнения.

На примере частного случая асимптотического приближения, описываемого уравнением диффузии, продемонстрировано, что использование экспоненциального распределения длины свободного пробега частиц тогда, когда оно таким не является, может приводить к значительной ошибке в получаемом решении.

Предложенная модель допускает следующие обобщения. Прямолинейное движение может сменяться не только броуновским, но и прямолинейным же, но в другом направлении. Это аналогично явлению внутриклеточного транспорта, когда везикула перескакивает с одной микротрубочки на другую. Частицы могут поглощаться (деградировать), и могут иметься источники частиц.

Модель может быть представлена также в криволинейных координатах, например в сферических. Это будет аналогично внутриклеточному транспорту грузов по цитоскелету.

Работа была поддержана грантом РФФИ № 17-01-00638a.

\section{Список литературы}

[1] Ross J.L., Ali M.Y., Warshaw D.M. // Curr. Opin. Cell Biol. 2008. Vol. 20. P. 41-47.

[2] Benichou O., Loverdo C., Moreau M., Voituriez R. // Rev. Mod. Phys. 2011. Vol. 83. P. 81-129.

[3] Viswanathan G.M., Da Luz M.G.E., Raposo E.P., Stanley H.E. The Physics of Foraging: An Introduction to Random Searches and Biological Encounters. Cambridge: University Press, Cambridge, 2011.
[4] Smith D.A., Simmons R.M. // Biophys. J. 2001. Vol. 80. P. 45 68.

[5] Dinh A.-T., Theofanous T., Mitragotri S. // Biophys. J. 2005. Vol. 89. P. 1574-1588.

[6] Holcman D. // J. Stat. Phys. 2007. Vol. 127. P. 471-494.

[7] Lagache T., Dauty E., Holcman D. // Phys. Rev. E. 2009. Vol. 79. P. 011921.

[8] Thiel F., Schimansky-Geier L., Sokolov I.M. // Phys. Rev. E. 2012. Vol. 86. P. 021117.

[9] Bressloff P.C., Newby J.M. // Rev. Mod. Phys. 2013. Vol. 85. P. $135-196$

[10] Bressloff P.C. Stochastic Processes in Cell Biology. Springer, Cham., 2014.

[11] Plank M.J., Auger-Methe M., Codling E.A. In: Dispersal, Individual Movement and Spatial Ecology: A Mathematical Perspective / Под ред. M.A. Lewis, P.K. Maini, S.V. Petrovskii. Berlin: Springer, 2013. P. 33-52.

[12] Balint S., Vilanova I.V., Alvarez I.S., Lakadamyali M. // Proc. Natl. Acad. Sci. USA. 2013. Vol. 110. P. 3375-3380.

[13] Breed G.A., Severns P.M., Edwards A.M. // J. Roy. Soc. Interface. 2014. Vol. 12. N 103. P. 20140927.

[14] Feller $W$. An Introduction to Probability Theory and Its Applications. Vol. 2. N.Y:: Wiley, 1971.

[15] Ross S.M. Introduction to Probability Models. Amsterdam: Elssevier, 2007.

[16] Alt W. // J. Math. Biol. 1980. Vol. 9. P. 147-177.

[17] Rukolaine S.A. // Physica A. 2016. Vol. 450. P. 205-216.

[18] Bressloff P.C., Newby J.M. // Phys. Rev. E. 2011. Vol. 83. P. 061139.

[19] Hillen T. // Eur. J. Appl. Math. 2003. Vol. 14. P. 613-636.

[20] Hillen T., Painter K.J. In: Dispersal, Individual Movement and Spatial Ecology: A Mathematical Perspective / Ed. by M.A. Lewis, P.K. Maini, S.V. Petrovskii. Berlin: Springer, 2013. P. 177-222. 